\title{
Adverse Event End Day
}

National Cancer Institute

\section{Source}

National Cancer Institute. Adverse Event End Day. NCI Thesaurus. Code C83202.

The day of the week that an adverse event concluded. 